\title{
Editorial \\ Hidden Opportunities in Cytoreductive Surgery for Peritoneal Carcinomatosis of Colorectal Origin
}

\author{
George J. Chang, MD, MS and Laura A. Lambert, MD \\ Department of Surgical Oncology, Unit 444, The University of Texas, M. D. Anderson Cancer Center, 1515 Holcombe Blvd,
} Houston, TX 77230-1402, USA

The optimal management of peritoneal carcinomatosis of colorectal origin remains a challenging clinical problem characterized by more questions than answers. In recent years there has been a great expansion of therapeutic options for patients with metastatic colorectal cancer. Newer and more effective systemic chemotherapeutic agents (e.g., oxaliplatin and irinotecan), targeted biologic therapies (e.g., bevacizumab, cetuximab, panitumumab) and advances in surgical indications for resection of visceral metastases have greatly improved survival outcomes. However, for the more than $15 \%$ of patients with colorectal cancer who develop peritoneal metastases, data to guide their therapy are limited, often resulting in treatment decisions based upon strong patient and provider convictions rather than established experimental evidence.

Fortunately, significant efforts to improve the poor prognosis for these patients are ongoing. One of the most notable efforts is increased use of the combination treatment of cytoreductive surgery (CS) with perioperative (intra- or postoperative) intraperitoneal chemotherapy (PIC) with or without hyperthermia. Using this approach, single-center reports have noted 5 -year survival rates of $20 \%$ to over $40 \%$ for patients treated with CS and hyperthermic intraperitoneal chemotherapy (HIPEC) - twice the expected survival with systemic 5-fluorouracil chemotherapy alone. ${ }^{1-3}$ Consequently, these and other data have led to a recent consensus statement advocating the use of CS

Published online September 12, 2008.

Address correspondence and reprint requests to: George J. Chang, MD, MS; E-mail: gchang@mdanderson.org

Published by Springer Science+Business Media, LLC @ 2008 The Society of Surgical Oncology, Inc. and PIC in patients with peritoneal carcinomatosis from colorectal cancer. ${ }^{4}$

In this issue Franko and colleagues report a retrospective analysis of prospectively collected data from their single-institutional experience regarding multivisceral resection (MVR) during the course of $\mathrm{CS}$ and HIPEC for colorectal peritoneal carcinomatosis $(n=65) .{ }^{5}$ The purpose of their study was to evaluate the impact of MVR during CS and HIPEC on morbidity and survival outcomes. The overall morbidity rate in this study was $60 \%$. The performance of a bowel anastomosis was an important identified risk factor of significant increase in morbidity $(70 \%$ with anastomosis versus $33 \%$ without, $P=0.003$ ) including increased rates of reoperation, wound complications, enterocutaneous fistulae, and prolonged ileus. MVR, defined as the resection of two or more organs beyond peritonectomy and omentectomy, was performed in 35 of 65 patients $(54 \%)$. MVR was not associated with significant differences in perioperative morbidity or mortality compared with patients who did not require MVR during CS. Additionally, patients who underwent complete or nearly complete cytoreduction (defined as residual tumor implants $<5 \mathrm{~mm}$ ) had significantly better median survival after CS and HIPEC compared with patients who had an incomplete cytoreduction (20.2 versus 10.6 months, $P<0.001)$ The performance of MVR did not affect median survival after CS and HIPEC - neither from the time of diagnosis of stage IV disease nor from the time of CS and HIPEC.

At least two important messages are highlighted by the authors' findings. Firstly, peritoneal cytoreduction with HIPEC is a potentially effective modality for selected patients with colorectal-cancer-associated peritoneal carcinomatosis. Secondly, the key to 
maximizing survival outcomes is the achievement of a complete cytoreduction, even when requiring MVR, analogous to strategies for management of metastases to other sites.

However, the data presented in this article also highlight a number of unresolved issues regarding the role of CS and HIPEC in the management of colorectal peritoneal carcinomatosis. Cytoreductive surgery and HIPEC are associated with high morbidity (60\% in this study and $23-44 \%$ in a number of other studies), making optimal patient selection one of the most important unresolved issues. ${ }^{2,6-10}$ Although a novel finding of this study was the negative survival impact of the presence of carcinomatosis on computed tomography (CT) scan, further elaboration as to how patients without radiographic evidence were identified for CS and HIPEC would aid in patient selection. It is reasonable to assume that identifying patients earlier (i.e., with less tumor burden) could lower the extent and morbidity of CS and HIPEC, increase the rate of complete cytoreduction, and improve the survival benefit.

Another unresolved issue is the definition of an optimal cytoreduction-how much is enough? In their analysis the authors used a residual implant size cutoff of $5 \mathrm{~mm}$ while others have used $2.5 \mathrm{~mm} .{ }^{4,11}$ It is likely that the optimal level will be influenced by a variety of factors such as tumor burden, disease distribution, prior surgical history, tumor biology, and the type of intraperitoneal chemotherapy used-all areas which require further investigation.

Perhaps the most important unanswered question highlighted by this and all other studies of CS and HIPEC to date is the extent to which each of the components of treatment (e.g., cytoreduction or intraperitoneal chemotherapy) improves outcomes over modern multi-agent systemic therapies. Unfortunately, answering this question is more complicated than simply comparing survival outcomes after CS and HIPEC with best systemic therapy. Given the relatively high risk of morbidity and mortality associated with CS and HIPEC, any potential advantage needs to be measured in terms of both survival benefit and treatment toxicity. In addition, because CS and HIPEC combine two treatment modalities, the effects of each modality (both benefit and harm) must be separately examined.

Undeniably, one of the most predictive factors for survival among patients undergoing CS and HIPEC for peritoneal carcinomatosis is completeness of cytoreduction. Franko et al. have clearly demonstrated that complete cytoreduction is the key to maximizing survival outcomes after CS and HIPEC for colorectal carcinomatosis. Additionally the finding of an absolute increase in survival associated with MVR (32.8 versus 20.2 months, $P=0.787$ ) is intriguing (despite this difference lacking statistical significance), perhaps reflecting a more complete cytoreduction in MVR patients. A recent pooled analysis from 28 centers, which included 506 patients who had CS and PIC, demonstrated that completeness of cytoreduction was the principal prognostic variable in a multivariate Cox regression model. Median survival rates were as high as 32.4 months following complete cytoreduction (no macroscopic residual carcinomatosis), 24 months following near-complete cytoreduction (residual carcinomatosis nodules $<5 \mathrm{~mm}$ ), and only 8.4 months following incomplete reduction (residual carcinomatosis nodules $>5 \mathrm{~mm})(P<0.0001){ }^{7}$ In a separate randomized comparison of CS and HIPEC followed by systemic chemotherapy versus systemic chemotherapy and supportive surgery alone, median overall survival rate with complete resection has been reported to be as high as 48 months versus less than 10 months when gross disease remained. ${ }^{3}$

There is also evidence that complete cytoreduction (without PIC) in conjunction with systemic chemotherapy may confer a measurable benefit for patients with colorectal peritoneal metastases. A randomized trial compared cytoreductive surgery with early PIC followed by systemic chemotherapy versus CS followed by systemic chemotherapy alone. Unfortunately, due to strong patient preferences, this trial closed prematurely after enrolling only 35 of a total 90 planned patients. An unexpected but very important finding was the outstanding survival rate of $60 \%$ at 2 years for either arm of the trial. Equally as important was the observation that the PIC arm experienced a higher rate of perioperative morbidity and mortality that likely would have been statistically significant if the trial were to have completed its accrual $(69 \%$ versus $37 \%, P=0.092)$. In addition, fewer patients in the PIC arm subsequently received postoperative systemic chemotherapy as scheduled (56\% versus $100 \%, P=0.0017$ ), perhaps as a result of increased rates of morbidity and mortality. ${ }^{1}$

Although the benefit of complete cytoreduction is well demonstrated, the added benefit of intraperitoneal chemotherapy is not as clearly defined. An analysis of the distribution, timing, and outcomes of recurrent disease after complete or near-complete cytoreduction and PIC for peritoneal carcinomatosis from colorectal cancer was recently reported. ${ }^{11}$ This analysis included 70 patients with adequate cytoreduction (defined as $<2.5 \mathrm{~mm}$ ) out of a carefully selected 156 attempted CS (58\%). Despite optimal 
patient selection and aggressive CS, 49 patients $(78 \%)$ developed disease recurrence at a median time of 9 months. In 21 patients $(43 \%)$ disease recurrence included distant metastases. Such high rates of treatment failure (including distant metastases) despite careful patient selection and complete CS with HIPEC are the background against which the high rates of morbidity and relatively short life expectancy of patients with colorectal carcinomatosis must be viewed. We must consider these and other potential consequences of HIPEC (prolonged hospital stays, increased length of time to complete recovery, and decreased perioperative quality of life) in order to make an evidenced-based recommendation regarding its use. ${ }^{12}$ Furthermore, recent data reporting median survival rates of up to 31.8 months among patients with unresectable stage IV colorectal cancers receiving a maintenance regimen of the targeted anti-vascular endothelial growth factor (VEGF) antibody, bevacizumab, in combination with chemotherapy make it imperative to determine the independent roles of both CS and HIPEC in the context of the continual evolution of more efficacious systemic therapy. ${ }^{13}$

Yet perhaps in these data are the hidden opportunities for the treatment of peritoneal carcinomatosis of colorectal origin. This study by Franko et al. provides additional support of the rationale for systematic investigation of the independent roles of CS and HIPEC in the context of modern systemic therapy. Improvement in our understanding of the peritoneum as a resectable organ combined with advanced intraperitoneal cytoreductive techniques may now allow us to approach carcinomatosis as any other site of resectable visceral metastasis. In addition, the improved efficacy of systemic chemotherapy offers a potential opportunity to employ cytoreductive surgery as a component of multimodality curative therapy. Proper patient selection, including referral of patients with peritoneal carcinomatosis prior to exhaustion of systemic therapeutic options, is essential. In determining the benefit of CS alone, without HIPEC, arises an opportunity to evaluate the incremental benefit of HIPEC. Determining the potential benefit of complete cytoreduction as an adjuvant to best systemic therapy is both the rational and essential next step.

\section{REFERENCES}

1. Elias D, Blot F, El Otmany A, et al. Curative treatment of peritoneal carcinomatosis arising from colorectal cancer by complete resection and intraperitoneal chemotherapy. Cancer 2001; 92:71-6.

2. Elias D, Raynard B, Farkhondeh F, et al. Peritoneal carcinomatosis of colorectal origin. Gastroenterol Clin Biol 2006; 30:1200-4.

3. Verwaal VJ, Bruin S, Boot H, et al. 8-Year follow-up of randomized trial: cytoreduction and hyperthermic intraperitoneal chemotherapy versus systemic chemotherapy in patients with peritoneal carcinomatosis of colorectal cancer. Ann Surg Oncol 2008; 15:2426-32.

4. Esquivel J, Sticca R, Sugarbaker P, et al. Cytoreductive surgery and hyperthermic intraperitoneal chemotherapy in the management of peritoneal surface malignancies of colonic origin: a consensus statement. Society of Surgical Oncology. Ann Surg Oncol 2007; 14:128-33.

5. Franko J, Gusani N, Holtzman M, et al. Multivisceral resection does not affect morbidity and survival after cytoreductive surgery and chemoperfusion for carcinomatosis from colorectal cancer. Ann Surg Oncol 2008. DOI: 10.1245/s10434-0080105-x.

6. Glehen O, Cotte E, Schreiber V, et al. Intraperitoneal chemohyperthermia and attempted cytoreductive surgery in patients with peritoneal carcinomatosis of colorectal origin. Br J Surg 2004; 91:747-54.

7. Glehen O, Kwiatkowski F, Sugarbaker PH, et al. Cytoreductive surgery combined with perioperative intraperitoneal chemotherapy for the management of peritoneal carcinomatosis from colorectal cancer: a multi-institutional study. J Clin Oncol 2004; 22:3284-92.

8. Shen P, Hawksworth J, Lovato J, et al. Cytoreductive surgery and intraperitoneal hyperthermic chemotherapy with mitomycin $\mathrm{C}$ for peritoneal carcinomatosis from nonappendiceal colorectal carcinoma. Ann Surg Oncol 2004; 11:178-86.

9. Pilati P, Mocellin S, Rossi CR, et al. Cytoreductive surgery combined with hyperthermic intraperitoneal intraoperative chemotherapy for peritoneal carcinomatosis arising from colon adenocarcinoma. Ann Surg Oncol 2003; 10:508-13.

10. Kecmanovic DM, Pavlov MJ, Ceranic MS, et al. Treatment of peritoneal carcinomatosis from colorectal cancer by cytoreductive surgery and hyperthermic perioperative intraperitoneal chemotherapy. Eur J Surg Oncol 2005; 31:147-52.

11. Bijelic L, Yan TD, Sugarbaker PH. Failure analysis of recurrent disease following complete cytoreduction and perioperative intraperitoneal chemotherapy in patients with peritoneal carcinomatosis from colorectal cancer. Ann Surg Oncol 2007; 14:2281-8.

12. McQuellon RP, Danhauer SC, Russell GB, et al. Monitoring health outcomes following cytoreductive surgery plus intraperitoneal hyperthermic chemotherapy for peritoneal carcinomatosis. Ann Surg Oncol 2007; 14:1105-13.

13. Grothey A, Sugrue M, Purdie D, et al. Association between exposure to bevacizumab (BV) beyond first progression (BBP) and overall survival (OS) in patients (pts) with metastatic colorectal cancer (mCRC): Results from a large observational study (BRiTE). American Society of Clinical Oncology Annual Meeting. Chicago, IL, 2007. 